\title{
Optimal Environmental Road pricing
}

\author{
Working paper 168 \\ Olof Johansson-Stenman \\ Department of Economics, Göteborg University
}

\begin{abstract}
An optimal first-best road charge should not only be differentiated with respect to factors that affect the direct external environmental and time costs from the road-user himself. Indirect effects, such as the fact that other cars will be more polluting when congestion increases, should also be taken into account.
\end{abstract}

\section{Introduction}

Road pricing from an economic point of view has largely been motivated by the time costs that each road user imposes on other road users, since the classical discussion of external costs by Pigou (1920). But in recent years the discussion has also considered other externalities as well, with particular attention being given to local air-pollution problems associated with urban transportation. The overall objective of this paper is to derive analytical expressions of how to take environmental concern into account in optimal steady-state road pricing models in the tradition of Walters (1961), Newbery (1988, 1990) and De Meza and Gould (1987). Special emphasis is given to indirect environmental costs, which are typically overlooked. 


\section{The Model}

A representative individual's utility depends positively on his own travelled distance per time unit (or flow) $q$, the environmental quality $E$, leisure $l$, and private consumption of a composite $\operatorname{good} x$ :

$$
U=u(q, E, l, x)
$$

For analytic simplicity, and without loss of generality, we start by deriving the optimum conditions for any given amount of work $L$ (e.g. at the optimal level at the overall system optimum). The government's objective is to maximize utility for the representative individual subject to a time constraint and a resource constraint. The time constraint is given by

$$
l=\hat{t}-L-\frac{q}{v}
$$

where $\hat{t}$ is the total time available (24h/day), $L$ is the amount to work, $v$ is the endogenously determined speed, and $q / v$ is travel time (i.e. time spent on travelling). Hence, leisure is total time minus time used for working and travelling. The overall budget or resource constraint is given by

$$
x+z q=w L
$$

where $z$ is the private travel cost per distance unit, and $w$ is the exogenously given wage per time unit. Hence, total consumption on travel and other goods is equal to the wage per hour times the number of working hours. Private travel cost $z$ increases with the degree of congestion (and hence decreases with the average speed in the relevant interval) due to increased wear and tear and fuel consumption per $\mathrm{km}$ :

$$
z=z(v),
$$


where $\frac{\mathrm{d} z}{\mathrm{~d} v}<0$. Environmental damage from travelling per time unit is given by the product of the distance travelled per time unit $q$, emissions per distance unit $\sigma$ (denoted emission factor), and exposure $b$, i.e. the fraction of the emission that is inhaled by people (or reaches any other target):

$$
E=q \sigma b .
$$

The emission factor (which may be seen as a vector reflecting many different emissions such as $\mathrm{NO}_{\mathrm{x}}, \mathrm{pm}_{2.5}$ etc.), in turn, often varies drastically with the average speed, reflecting more starts, stops and accelerations when the speed is lower:

$$
\sigma=\sigma(v)
$$

where $\frac{\mathrm{d} \sigma}{\mathrm{d} v}<0$. The exposure $b$ depends on the population density close to the vehicles where the emissions occur, $\delta$ :

$$
b=b(\delta) \text {, }
$$

where $\frac{\mathrm{d} b}{\mathrm{~d} \delta}>0$. The fact that the population density also varies with congestion and average speed may not be equally apparent. Nevertheless, a large fraction of the health effects from car emissions are caused to other road-users who are close to the place of pollution, and when congestion increases and speed decreases, the vehicles come closer together. Hence, we can write:

$$
\delta=\delta(v),
$$

where $\frac{\mathrm{d} \delta}{\mathrm{d} v}<0$. Speed, in turn, is given by a speed-flow relationship:

$$
v=v(q),
$$


where $\frac{\mathrm{d} v}{\mathrm{~d} q}<0$. To obtain the socially optimal amount of travel we first substitute (2)-(9) into (1), to obtain:

$$
U=u(q, q \sigma(v(q)) b(\delta(v(q)), \hat{\imath}-L-q /(v(q)), w L-z(v(q)) q) .
$$

Now utility is expressed solely as a function of $q$, for a given amount of work $L$. The first order condition of (10) with respect to $q$ then gives:

$$
\begin{aligned}
& \frac{\partial u}{\partial q}+\frac{\partial u}{\partial E}\left(\sigma b+q \frac{\mathrm{d} \sigma}{\mathrm{d} v} \frac{\mathrm{d} v}{\mathrm{~d} q} b+q \sigma \frac{\mathrm{d} b}{\mathrm{~d} \delta} \frac{\mathrm{d} \delta}{\mathrm{d} v} \frac{\mathrm{d} v}{\mathrm{~d} q}\right)- \\
& \frac{\partial u}{\partial l}\left(\frac{1}{v}-\frac{q}{v^{2}} \frac{\mathrm{d} v}{\mathrm{~d} q}\right)-\frac{\partial u}{\partial x}\left(\frac{\mathrm{d} z}{\mathrm{~d} v} \frac{\mathrm{d} v}{\mathrm{~d} q} q+z\right)=0
\end{aligned}
$$

Assuming a non-cooperative Nash equilibrium, a utility-maximizing individual disregards the external costs caused to others, and hence treats $E$ and $v$ as given, but takes into account a road charge per distance $\tau$ imposed by the government. Any net revenues are assumed to be distributed back in a lump-sum manner. Hence, the private budget is given by

$$
x+(z(v)+\tau) q=w L+\tau^{0},
$$

where $\tau^{0}$ is a lump-sum transfer. The individual will consequently maximise the following expression:

$$
\left.U=u(q, \bar{E}, \tau-L-q / \bar{v}), w L+\tau^{0}-z(\bar{v}) q-\tau q\right),
$$

where a bar denotes that the value of the variable is considered constant by the individual. The private optimum condition with respect to $q$ is consequently given by:

$$
\frac{\partial u}{\partial q}-\frac{\partial u}{\partial l} \frac{1}{v}-\frac{\partial u}{\partial x}(z+\tau)=0
$$

We can then combine the social and the private optimum conditions, i.e. (11) and (14), and solve for the optimal road charge $\tau$ : 


$$
\begin{aligned}
& \tau=-\frac{\partial u / \partial l}{\partial u / \partial x} \frac{q}{v^{2}} \frac{\mathrm{d} v}{\mathrm{~d} q}+q \frac{\mathrm{d} z}{\mathrm{~d} v} \frac{\mathrm{d} v}{\mathrm{~d} q} \\
& +M R S_{E x}\left(\sigma b+q \frac{\mathrm{d} \sigma}{\mathrm{d} v} \frac{\mathrm{d} v}{\mathrm{~d} q} b+q \sigma \frac{\mathrm{d} b}{\mathrm{~d} \delta} \frac{\mathrm{d} \delta}{\mathrm{d} v} \frac{\mathrm{d} v}{\mathrm{~d} q}\right)
\end{aligned}
$$

where $M R S_{E x}=\frac{\partial u / \partial E}{\partial u / \partial x}$, or the marginal willingness to pay for increased environmental quality in terms of $x$. The optimal charge can thus be divided into five terms: The first term reflects the time costs that each road user, on the margin, imposes on other road users, whereas the second term reflects others' increased private costs per $\mathrm{km}$, in terms of fuel consumption and wear and tear, which the additional congestion due to an additional vehicle $\mathrm{km}$ causes. The third term reflects the marginal damage of pollution from each vehicle per unit of distance. This term naturally varies with the traffic intensity, as reflected by the speed, since both the emission factors as well as the surrounding local population density vary with speed.

The fourth and the fifth terms may be more surprising. The fourth term reflects the fact that other vehicles will become more polluting with each extra $\mathrm{km}$ driven. ${ }^{1}$ One may have thought that this effect would be a pecuniary externality, and hence not price-relevant, since the other road users will pay for their emissions too; hence they will pay for the additional pollution that is caused by the additional congestion by another vehicle. To intuitively explain why it is a real price-relevant externality, consider each welfare component separately, and let us for simplicity neglect other externalities (e.g. others' time costs) for a moment. An extra km by Alice will cause other cars, including Bob's car, to slow down, and hence to pollute more per $\mathrm{km}$. These increased emissions will cause a cost to the rest of society, including Carl who is not a road user. But at the optimum, others including Bob will pay an equally large charge to the government, or to the rest of the society, to correct for this increased externality. Hence, Carl and 
the rest of the society are indifferent to whether Alice drives an extra km, as long as Alice, Bob and others pay for their increased emissions. However, the other road-users, such as Bob, are not indifferent, since an additional $\mathrm{km}$ by Alice implies that they will have to pay larger emission charges. This is the social cost that the fourth term of (15) reflects.

The fifth term similarly reflects that the congestion increase, and the corresponding speed decrease, of an additional vehicle $\mathrm{km}$ cause the local population density to increase, which increases the exposure per emission unit, implying that others' emissions become more damaging. Consequently, other road users will have to pay correspondingly higher road charges, and the motivation behind this term is thus analogous to the one behind the fourth term.

Moreover, if the amount of work is endogenous, and chosen optimally by the individual, we can differentiate (13) with respect to $L$ and obtain:

$$
\frac{\partial u / \partial l}{\partial u / \partial x}=w
$$

Hence, the shadow price of leisure, and hence of time generally, is equal to the wage $w$, which follows intuition. We can then simplify (15) further to obtain

$$
\begin{aligned}
& \tau=-w \frac{q}{v^{2}} \frac{\mathrm{d} v}{\mathrm{~d} q}+q \frac{\mathrm{d} z}{\mathrm{~d} v} \frac{\mathrm{d} v}{\mathrm{~d} q} \\
& +\operatorname{MRS}_{E x}\left(\sigma b+q \frac{\mathrm{d} \sigma}{\mathrm{d} v} \frac{\mathrm{d} v}{\mathrm{~d} q} b+q \sigma \frac{\mathrm{d} b}{\mathrm{~d} \delta} \frac{\mathrm{d} \delta}{\mathrm{d} v} \frac{\mathrm{d} v}{\mathrm{~d} q}\right)
\end{aligned}
$$

However, it is of course possible that an individual considers the disutility of one more hour of work to be different from that of one more hour of travel time. If working is more pleasurable (such as for academic researchers, right?), then the appropriate shadow value exceeds $w$, and vice versa. Most empirical studies seem to conclude that the private value of time is on average somewhat lower than the wage; see e.g. Calfee and Winston (1998). In reality there are of course

\footnotetext{
${ }^{1}$ Johansson (1997) presents simulation evidence that these effects can sometimes be substantial.
} 
also many distortions such as income taxes that may cause deviations. Nevertheless, the qualitative conclusions implied by (15) hold, irrespective of whether the value of time is constant or not.

\section{Conclusions}

This paper has used a stylised model to derive a first-best road charge with respect to different possible components. Although the model captures nothing of the large heterogeneity over time, in space and between people that are essential to any road pricing system in practice, it is hard to see why the main conclusions in this paper would be dependent on these simplifying assumptions. It was shown that not only should road users pay for the direct time and environmental costs that they impose on other road users and other people; they should also pay a charge corresponding to the increase in others' fuel costs, wear-and-tear costs. Moreover, they should also pay for the increase in others' environmental charges, since other cars will be more polluting, and their damage per emission unit will be higher due to increased population density, when the traffic increases. As far as I know, this has never been shown in a utility-theoretic model before. One can of course argue that practical road-pricing systems must reflect tradeoffs between allocative efficiency and simplicity, and can hence not take into account all theoretical subtleties. It is nevertheless valuable to know the theoretical benchmark solution before making all necessary simplifications.

\section{References}

Calfee and Winston (1998), "The value of automobile travel time: Implications for congestion policy", Journal of Public Economics, 69, 83-102.

De Meza, D. and J. R. Gould (1987) "Free Access versus Private Property in a Resource: Income Distributions Compared", Journal of Political Economy, 95, 1317-15. 
Johansson, O. (1997), “Optimal road-pricing: simultaneous treatment of time losses, increased fuel consumption, and emissions." Transportation Research D, 2(2), 77-87.

Newbery, D. M. (1988), “Road user charges in Britain.” Economic Journal, 98, 161-76.

Newbery, D. M. (1990), "Pricing and congestion: economic principles relevant to pricing roads." Oxford Review of Economic Policy, 6(2), 22-39.

Pigou, A.C. (1920), The Economics of Welfare, 2nd edition, AMS Press.

Walters A.A. (1961), “The theory and measurement of private and social cost of highway congestion", Econometrica, 29: 676-697. 